\title{
Magnetic excitations and structure of the topological semimetal $\mathrm{YbMnSb}_{2}$
}

\author{
S. M. Tobin ${ }^{1}$, J-R. Soh ${ }^{2}$, H. Su ${ }^{3}$, B. Ouladdiaf ${ }^{4}$, A. Piovano ${ }^{4}$, Y-F. Guo ${ }^{3}$, D. Prabhakaran ${ }^{1}$, A. T. Boothroyd ${ }^{1}$ \\ ${ }^{1}$ Department of Physics, University of Oxford, United Kingdom, ${ }^{2}$ Institute of Physics, École Polytechnique Fédéral de Lausanne, \\ Switzerland, ${ }^{3}$ School of Physical Science and Technology, ShanghaiTech University, China, ${ }^{4}$ Institut Laue Langevin (ILL), France
}

siobhan.tobin@physics.ox.ac.uk

Topological semimetals have high carrier mobility in the form of quasiparticles resembling relativistic fermions. Experimental realisations of magnetic topological semimetals are relatively thin on the ground. Here we probe both the magnetic structure and interactions of the topological semimetal candidate $\mathrm{YbMnSb}_{2}$ using neutron scattering.

$\mathrm{YbMnSb}_{2}$ belongs to the $P 4 / \mathrm{nmm}$ space group and shows evidence of a magnetic ordering transition involving the Mn moments at $\sim 350 \mathrm{~K}$ [1]. This is a relatively high Néel temperature among the family of materials $A \mathrm{MnSb}_{2}(A=\mathrm{Ca}, \mathrm{Sr}, \mathrm{Ba}, \mathrm{Yb}, \mathrm{Eu})$, which has demonstrated characteristics of the topological semimetals. The quasi-2D plane formed by the Sb 'square' may host Weyl or Dirac fermions [1-3]. $\mathrm{YbMnSb}_{2}$ has previously been studied via quantum oscillations, magnetometry, optical spectroscopy, ab initio band structure calculations, and angle-resolved photon emission spectroscopy $[1,4,5]$. Interestingly, these studies reached different conclusions as to the magnetic structure of $\mathrm{YbMnSb}_{2}$, and hence its semimetal nature: the jury is out on whether it is a Dirac [4], nodal-line [5], or Weyl semimetal [1].

In this presentation I shall report the magnetic structure of $\mathrm{YbMnSb}_{2}$ found by neutron diffraction, which is different to any previously proposed structures: C-type antiferromagnetism with the spins pointing along the $c$ axis. This magnetic structure is shared by $\mathrm{YbMnBi}_{2}$ [6]. Dirac physics is also seen in such $A \mathrm{MnBi}_{2}$ materials; however, Bi rather than Sb layers results in stronger spin-orbit coupling. This widens the band gap at any nodes and makes the resulting quasiparticles more massive [1]. We have also measured the spin wave spectrum of $\mathrm{YbMnSb}_{2}$ and the results of this measurement will be described and compared with the spin dynamics in related materials. The implications for the topology of the electrons will be discussed.
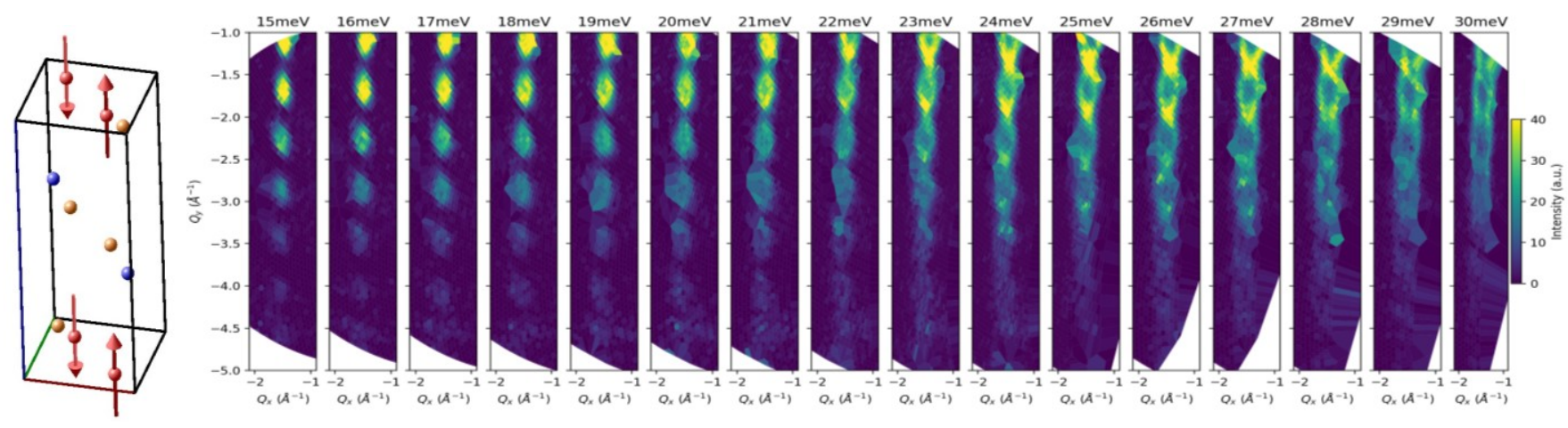

Figure 1. left, proposed magnetic structure of $\mathrm{YbMnSb}_{2}$; right, spin wave spectrum of $\mathrm{YbMnSb}_{2}$ in the $h 0 l$ plane obtained from inelastic neutron scattering.

[1] Wang, Y.-Y., Xu, S., Sun, L.-L., and Xia, T.-L. Physical Review Materials 2(2), 021201 February (2018).

[2] Liu, J., Hu, J., Cao, H., Zhu, Y., Chuang, A., Graf, D., Adams, D. J., Radmanesh, S. M. A., Spinu, L., Chiorescu, I., and Mao, Z. Scientific Reports 6(1), 2016

[3] He, J. B., Fu, Y., Zhao, L. X., Liang, H., Chen, D., Leng, Y. M., Wang, X. M., Li, J., Zhang, S., Xue, M. Q., Li, C. H., Zhang, P., Ren, Z. A., and Chen, G. F. Physical Review B 95(4), 045128 January (2017).

[4] Kealhofer, R., Jang, S., Griffin, S. M., John, C., Benavides, K. A., Doyle, S., Helm, T., Moll, P. J. W., Neaton, J. B., Chan, J. Y., Denlinger, J. D., and Analytis, J. G. Physical Review B 97(4), 045109 January (2018).

[5] Qiu, Z., Le, C., Liao, Z., Xu, B., Yang, R., Hu, J., Dai, Y., and Qiu, X. Physical Review B 100(12), 125136 September (2019).

[6] Soh, J.-R., Jacobsen, H., Ouladdiaf, B., Ivanov, A., Piovano, A., Tejsner, T., Feng, Z., Wang, H., Su, H., Guo, Y., Shi, Y., and Boothroyd, A. T. Physical Review B 100(14), 144431 October (2019).

Keywords: topological semimetals, quasiparticles, magnetic structures, $\mathrm{YbMnSb}_{2}$ 